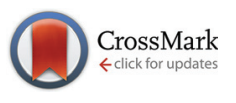

Cite this: Food Funct., 2017, 8, 975

\section{Contribution of the phenolic composition to the antioxidant, anti-inflammatory and antitumor potential of Equisetum giganteum L. and Tilia platyphyllos Scop.}

\author{
Inés Jabeur, ${ }^{\text {a,b }}$ Natália Martins, ${ }^{a}$ Lillian Barros, ${ }^{\text {aa,c }}$ Ricardo C. Calhelha, ${ }^{a}$ Josiana Vaz, ${ }^{a}$ \\ Lotfi Achour, ${ }^{b}$ Celestino Santos-Buelga ${ }^{d}$ and Isabel C. F. R. Ferreira*a
}

Received 11th December 2016, Accepted 18th January 2017

DOI: 10.1039/c6fo01778a

rsc.li/food-function
Naturally-occurring phytochemicals have received pivotal attention in the last few years, due to the increasing evidence of biological activities. Thus, in the present study, the antioxidant, anti-inflammatory and antitumor potentials of hydroethanolic extracts rich in phenolic compounds obtained from Equisetum giganteum L. and Tilia platyphyllos Scop. were assessed and directly correlated with their content of phenolic compounds, by using HPLC-DAD-ESI/MS analysis. T. platyphyllos showed the higher bioactive potential, evaluated in terms of antioxidant (radical scavenging effects $-105 \mu \mathrm{g} \mathrm{mL}^{-1}$, reducing power - $123 \mu \mathrm{g} \mathrm{mL}^{-1}, \beta$-carotene bleaching inhibition - $167 \mu \mathrm{g} \mathrm{mL}^{-1}$, and lipid peroxidation inhibition $\left.56 \mu \mathrm{g} \mathrm{mL}{ }^{-1}\right)$, anti-inflammatory $\left(225 \mu \mathrm{g} \mathrm{mL}^{-1}\right.$ inhibited $50 \%$ of nitric oxide production) and antitumor (breast - $224 \mu \mathrm{g} \mathrm{mL}{ }^{-1}$; lung - $247 \mu \mathrm{g} \mathrm{mL}^{-1}$; cervical - $195 \mu \mathrm{g} \mathrm{mL} \mathrm{L}^{-1}$ and hepatocellular - $173 \mu \mathrm{g} \mathrm{mL}{ }^{-1}$ carcinoma cells) activity, without having cytotoxic effects $\left(>400 \mu \mathrm{g} \mathrm{mL}^{-1}\right)$. These biological properties were positively correlated with its content and composition of phenolic compounds. Flavonoid contents were markedly higher than the content of phenolic acids, in both samples, being respectively $50.4 \mathrm{mg} \mathrm{g}^{-1}$ and $11.65 \mathrm{mg} \mathrm{g}^{-1}$ for T. platyphyllos, and $21.7 \mathrm{mg} \mathrm{g}^{-1}$ and $4.98 \mathrm{mg} \mathrm{g}^{-1}$ for E. giganteum. Moreover, while in E. giganteum extract, kaempferol-O-glucoside-O-rutinoside was the most abundant flavonoid, in T. platyphyllos extract protocatechuic acid and (-)-epicatechin were the most abundant phenolic acid and flavonoid, respectively. In relation to their content of phenolic acids, protocatechuic and caffeic acids existed in higher abundance in T. platyphyllos and E. giganteum hydroethanolic extracts, respectively. However, it would be interesting to evaluate the in vivo efficacy of both plant extracts to unveil the involved modes of action and to establish effective therapeutic doses.

\section{Introduction}

Natural products, among them plant-derived preparations, revealed to be a rich source of bioactive compounds, such as alkaloids, terpenes, steroids and phenolic compounds, are substances responsible for multiple biological effects. ${ }^{1-8}$

\footnotetext{
${ }^{a}$ Mountain Research Centre (CIMO), ESA, Polytechnic Institute of Bragança, Campus de Santa Apolónia, 1172, 5300-253 Bragança, Portugal. E-mail: iferreira@ipb.pt, lillian@iph.pt; Fax: +351-273-325405, +351-273-325405; Tel: +351-273-303219, +351-273-303903

${ }^{b}$ Laboratoire de Recherche "Bioressources": Biologie Intégrative \& Valorisation", Institut Supérieur de Biotechnologie de Monastir, Avenue Tahar Hadded, BP 74, 5000, Université de Monastir, Monastir, Tunisia

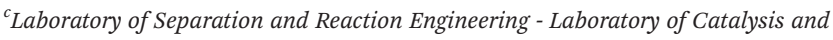
Materials (LSRE-LCM), Polytechnic Institute of Braganca,

Campus de Santa Apolónia, 1134, 5301-857 Bragança, Portugal

${ }^{d}$ Grupo de Investigación en Polifenoles (GIP-USAL), Facultad de Farmacia,

Universidad de Salamanca, Campus Miguel de Unamuno s/n, 37007 Salamanca, España
}

The latest scientific advances have shown that most of the bioactive phytochemicals are secondary metabolites from which through their consumption promissory phytopharmacological effects may be reached. ${ }^{5-8}$ In the case of phenolic compounds, antioxidant, anti-diabetic, anti-inflammatory, antimicrobial, antitumor, anti-ageing, cardioprotective, hypolipidemic, neuroprotective and hepatoprotective effects have been observed. ${ }^{5,9-11}$ However, the botanical class, plant family, species and origin affect the final chemical composition and consequently the bioactivity of plant-derived preparations; cultivation conditions, harvesting time, storage and processing techniques, and extraction conditions also play a determinant role. ${ }^{12-14}$

Equisetum giganteum L., commonly known as "giant horsetail" (English), "cavalinha" (Portuguese), "cola de caballo" (Spanish), is a native plant from Central and South America that belongs to the Equisetaceae family. ${ }^{15}$ The herb of this plant is largely used in dietary supplements as a diuretic, 
hemostatic, anti-inflammatory and anti-rheumatic agent. ${ }^{16,17}$ To the author's best knowledge, no studies are available describing its antioxidant or antitumor effects, and only one study could be found regarding the in vivo anti-inflammatory potential of aqueous extracts derived from stems, through using antigen-induced arthritis. ${ }^{17}$ The phenolic composition of this plant has been described, including the presence of caffeic acid derivatives and flavonoids (mainly quercetin and kaempferol derivatives). ${ }^{15,18}$ However, it should be highlighted that different types and proportions of solvents (namely, ethanol:water) were used by the authors in the extraction procedures.

Tilia platyphyllos Scop., commonly known as "linden" or "lime" (English), "tília" (Portuguese) and "tilo" (Spanish) is a well-known plant species that belongs to the Malvaceae family, native to Central and Southern Europe, from which very interesting biological activities have also been observed. ${ }^{19}$ Diuretic, stomachic, antispasmodic, sedative, antioxidant, hepatoprotective and anti-neuralgic properties are some of them. ${ }^{19}$ In vitro ${ }^{20}$ and in vivo ${ }^{19}$ antioxidant activities of the aqueous extract obtained, respectively, from leaves and flowers were previously described, in the last case by using animal models with ethanol-induced oxidative stress. The most abundant phenolic compounds in this species have been referred to as flavonoids (namely quercetin and kaempferol derivatives). ${ }^{20,21}$ Once again, and on the same lines as E. giganteum, different types of solvents (namely, ethanol: water) were used by the authors in the extraction procedures, and therefore different classes and relative abundances of phenolic compounds present were determined.

Thus, the present study aims to correlate the phenolic composition of both the mentioned plant species with their antioxidant, anti-inflammatory and antitumor potential, evaluated using hydroethanolic extracts.

\section{Experimental section}

\subsection{Samples and preparation of hydroethanolic extracts}

Aerial parts (namely, leaves, flowers and inflorescences) of Equisetum giganteum L. and Tilia platyphyllos Scop. were provided by Cantinho das Aromáticas, organic farmers from Vila Nova de Gaia (Portugal). The dried samples were reduced to powder form and subjected to an extraction procedure at room temperature $\left(\approx 25{ }^{\circ} \mathrm{C}\right)$, in three independent experiments, as follows: $1 \mathrm{~g}$ of each sample was extracted under magnetic stirring for $1 \mathrm{~h}$ in the dark with $30 \mathrm{~mL}$ of ethanol:water $(80: 20, \mathrm{v} / \mathrm{v})$, filtered and re-extracted under the same conditions. Then, ethanol was removed using a rotary evaporator (Büchi R-210, Flawil, Switzerland), while water was removed by lyophilisation. Finally, stock solutions of each hydroethanolic extract were prepared: $5 \mathrm{mg} \mathrm{mL} \mathrm{m}^{-1}$ in ethanol/water $(80: 20, \mathrm{v} / \mathrm{v})$ for antioxidant activity assays, $8 \mathrm{mg} \mathrm{mL}^{-1}$ in water for antitumor and anti-inflammatory assays, from which several dilutions were prepared and $2.5 \mathrm{mg} \mathrm{mL} \mathrm{m}^{-1}$ in ethanol/water $(80: 20, \mathrm{v} / \mathrm{v})$ for phenolic compound analysis (the extract was filtered through a $0.45 \mu \mathrm{m}$ Whatman syringe filter and transferred to amber color HPLC vial).

\subsection{Analysis of phenolic compounds}

HPLC-DAD-ESI/MS ${ }^{n}$ analyses were performed using a HewlettPackard 1100 chromatograph (Hewlett-Packard 1100, Agilent Technologies, Santa Clara, CA, USA), with a diode array detector (DAD) coupled to an HP Chem Station (rev. A.05.04) dataprocessing station and connected via the cell outlet to a MS detector API 3200 Qtrap (Applied Biosystems, Darmstadt, Germany) through an ESI source and a triple quadrupole-ion trap mass analyzer that was controlled by the Analyst 5.1 software. Separation was achieved on a Waters Spherisorb S3 ODS- $2 \mathrm{C}_{18}(3 \mu \mathrm{m}, 4.6 \times 150 \mathrm{~mm})$ column at $35{ }^{\circ} \mathrm{C}$, using a gradient elution; identification and quantification of the phenolic compounds were performed as described in ref. 22. The chromatograms were recorded at several wavelengths, characteristic of different classes of polyphenols, such as $280 \mathrm{~nm}$ for phenolic acids, flavan-3-ols and flavanones, $330 \mathrm{~nm}$ for hydroxycinnamic acids and $370 \mathrm{~nm}$ for flavonols and flavones. The calibration curves for each available phenolic standard (5-100 $\mu \mathrm{g} \mathrm{mL} \mathrm{mL}^{-1}$, Extrasynthese, Genay, France) were constructed: $(+)$-catechin $\left(y=135 x+33 ; R^{2}=0.999 ;\right.$ LOD, $0.17 \mu \mathrm{g} \mathrm{mL}{ }^{-1}$; LOQ, $\left.0.72 \mu \mathrm{g} \mathrm{mL} \mathrm{m}^{-1}\right)$; caffeic acid $(y=359 x+488$; $R^{2}=0.998$; LOD, $0.19 \mu \mathrm{g} \mathrm{mL}{ }^{-1}$; LOQ, $0.65 \mu \mathrm{g} \mathrm{mL}{ }^{-1}$ ); chlorogenic acid $\left(y=304 x-248 ; R^{2}=1\right.$; LOD, $0.20 \mu \mathrm{g} \mathrm{mL}^{-1}$; LOQ, $\left.0.68 \mu \mathrm{g} \mathrm{mL} \mathrm{m}^{-1}\right)$; (-)-epicatechin $\left(y=163 x+40 ; R^{2}=0.999 ;\right.$ LOD,

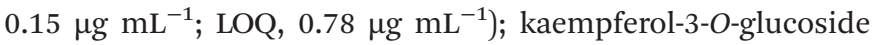
$\left(y=236 x+70 ; R^{2}=1\right.$; LOD, $0.11 \mu \mathrm{g} \mathrm{mL}{ }^{-1}$; LOQ, $\left.0.38 \mu \mathrm{g} \mathrm{mL}{ }^{-1}\right)$; kaempferol-3-O-rutinoside $\left(y=183 x+97 ; R^{2}=1\right.$; LOD, $0.13 \mu \mathrm{g} \mathrm{mL} \mathrm{m}^{-1}$; LOQ, $\left.0.43 \mu \mathrm{g} \mathrm{mL} \mathrm{m}^{-1}\right)$; naringenin $(y=540 x+161$; $R^{2}=0.994$; LOD, $0.20 \mu \mathrm{g} \mathrm{mL} \mathrm{m}^{-1}$; LOQ, $0.64 \mu \mathrm{g} \mathrm{mL} \mathrm{m}^{-1}$ ); protocatechuic acid $\left(y=258 x+328 ; R^{2}=0.998 ; \mathrm{LOD}, 0.14 \mu \mathrm{g} \mathrm{mL}^{-1}\right.$; LOQ, $\left.0.52 \mu \mathrm{g} \mathrm{mL}^{-1}\right)$; quercetin-3-O-glucoside $(y=336 x+358$; $R^{2}=0.998 ;$ LOD, $0.21 \mu \mathrm{g} \mathrm{mL} \mathrm{m}^{-1}$; LOQ, $0.71 \mu \mathrm{g} \mathrm{mL} \mathrm{m}^{-1}$ ) and quercetin-3-O-rutinoside $\left(y=281 x+374 ; R^{2}=0.998 ;\right.$ LOD, $0.22 \mu \mathrm{g} \mathrm{mL}{ }^{-1}$; LOQ, $0.75 \mu \mathrm{g} \mathrm{mL}{ }^{-1}$ ). The quantification of the peak areas was performed based on the UV signal and baseline to valley integration with the baseline projection mode and results were expressed as mg per $\mathrm{g}$ of the extract.

\subsection{Evaluation of the antioxidant activity}

Four different assays were used to assess the in vitro antioxidant potential of both plant extracts: scavenging effects on DPPH (2,2-diphenyl-1-picrylhydrazyl, Alfa Aesar, Ward Hill, MA, USA) radicals (RSA), reducing power measured by ferricyanide Prussian blue assay (RP), $\beta$-carotene bleaching inhibition (CBI) and lipid peroxidation inhibition (LPI), by thiobarbituric acid reactive substances (TBARS) assay, following a procedure previously described. ${ }^{23}$ All results obtained were expressed as $\mathrm{EC}_{50}$ values, corresponding to the extracts' concentration that provides $50 \%$ of antioxidant activity, or 0.5 of absorbance in the case of a reducing power assay. Three independent experiments were performed in each situation, for both plant extracts and Trolox (6-hydroxy-2,5,7,8-tetramethylchroman- 
2-carboxylic acid, Sigma-Aldrich, St Louis, MO, USA) was used as a positive control.

\subsection{Evaluation of the anti-inflammatory activity}

To assess the anti-inflammatory activity, a macrophage-like cell line RAW264.7 (European Collection of Animal Cell Culture, Salisbury, UK) was used and cultured, following a procedure previously described. ${ }^{24}$ The nitrite levels produced were determined by measuring the optical density at $515 \mathrm{~nm}$, in a microplate reader, and the obtained results were compared with the standard calibration curve. Dexamethasone $(50 \mu \mathrm{M}$, Sigma-Aldrich, St Louis, MO, USA) was used as the positive control. Three independent experiments were carried out and the final results were expressed in $\mathrm{EC}_{50}$ values, i.e. the extract concentration that inhibited $50 \%$ of the NO production.

\subsection{Evaluation of the cytotoxic and antitumor activity}

The cytotoxicity was evaluated using four tumor cell lines, HeLa (cervical carcinoma), HepG2 (hepatocellular carcinoma), MCF-7 (breast adenocarcinoma) and NCI-H460 (non-small cell lung cancer), using a protocol previously described, ${ }^{25}$ where the cell growth inhibition was measured at $540 \mathrm{~nm}$ (ELX800 microplate reader, Bio-Tek Instruments, Inc.; Winooski, VT, USA) using the sulforhodamine B assay (SRB, SigmaAldrich, St Louis, MO, USA).

For non-tumor porcine liver cells (PLP2) freshly harvested porcine liver was used in order to obtain the cell culture, designated as PLP2. The growth inhibition was evaluated using the SRB assay previously described. ${ }^{25}$

Three independent experiments were carried out for both assays, and the obtained results were expressed in $\mathrm{GI}_{50}$ values, i.e. the extract concentration that inhibited $50 \%$ of the net cell growth and ellipticine (Sigma-Aldrich, St Louis, MO, USA) was used as the positive control.

\subsection{Statistical analysis}

Three samples were used for each preparation and all the assays were carried out in triplicate. Results were expressed as mean values and standard deviation (SD) and analysis was performed through a Student's $t$-test with $p=0.05$, using a SPSS v. 23.0 program (IBM Corp., Armonk, New York, USA); a Pearson's correlation analysis was also carried out with a 95\% confidence level.

\section{Results and discussion}

\subsection{Detailed analysis of the phenolic composition}

Tables 1 and 2 present the phenolic compounds' characteristics, tentative identities and their relative abundance in E. giganteum and T. platyphyllos, respectively. The chromatographic profile of E. giganteum and T. platyphyllos can be observed in Fig. 1A and B. Equisetum giganteum presented fourteen phenolic compounds (two phenolic acids and twelve flavonol glycoside derivatives), while $T$. platyphyllos presented a more complex matrix regarding its phenolic composition, revealing the presence of thirty-three compounds (three phenolic acids, six flavan-3-ol derivatives, twenty flavonol glycoside derivatives, two flavanone and flavone derivatives). Compounds $2^{\mathrm{TP}}$ (protocatechuic acid), $4^{\mathrm{Eg}}$ (caffeic acid), $3^{\mathrm{Tp}}$ (catechin), $4^{\mathrm{Tp}}$ (5-O-caffeoylquinic acid), $6^{\mathrm{Tp}}$ (epicatechin), $15^{\mathrm{Tp}}$ (quercetin-3-O-rutinoside; rutin), $17^{\mathrm{Tp}}$ (quercetin-3-O-glucuronide), $10^{\mathrm{Eg}}$ and $20^{\mathrm{TP}}$ (quercetin-3-O-glucoside; isoquercitrin), $23^{\mathrm{TP}}$ and $11^{\mathrm{Eg}}$ (kaempferol-3-O-rutinoside) and $13^{\mathrm{Eg}}$ (kaempferol3-O-glucoside) were positively identified by comparison with authentic standards, as well as their MS fragmentation pattern, retention time and UV-vis characteristics. Kaempferol3-O-glucoside has been reported in hydroethanolic extracts of aerial parts of E. giganteum, ${ }^{15,16}$ and also as a quercetinhexoside. $^{16}$ Protocatechuic acid, catechin, epicatechin and

Table 1 Phenolic compounds' identification and quantification in the Equisetum giganteum extract

\begin{tabular}{|c|c|c|c|c|c|c|}
\hline Compound & $\begin{array}{l}R_{\mathrm{t}} \\
(\mathrm{min})\end{array}$ & $\begin{array}{l}\lambda_{\max } \\
(\mathrm{nm})\end{array}$ & $\begin{array}{l}\text { Molecular ion } \\
{[\mathrm{M}-\mathrm{H}]^{-}(\mathrm{m} / \mathrm{z})}\end{array}$ & $\mathrm{MS}^{2}(m / z)$ & Tentative identification & $\begin{array}{l}\text { Quantification } \\
\left(\mathrm{mg} \mathrm{g}^{-1} \text { extract) }\right.\end{array}$ \\
\hline $1^{\mathrm{Eg}}$ & 7.2 & 330 & 341 & $179(100)$ & Caffeic acid hexoside & $0.131 \pm 0.003$ \\
\hline $2^{\mathrm{Eg}}$ & 9.4 & 352 & 625 & $463(100), 301(32)$ & Quercetin-3,7-di-O-glucoside & $\operatorname{Tr}$ \\
\hline $3^{\mathrm{Eg}}$ & 11.5 & 348 & 755 & $593(100), 447$ (10), 285 (38) & Kaempferol-O-glucoside-O-rutinoside & $7.6 \pm 0.2$ \\
\hline $4^{\mathrm{Eg}}$ & 11.9 & 330 & 179 & $135(100)$ & Caffeic acid & $4.86 \pm 0.03$ \\
\hline $5^{\mathrm{Eg}}$ & 14.1 & 348 & 651 & $489(33), 447$ (17), 285 (17) & Kaempferol- $O$-acetylglucoside- $O$-glucoside & $0.087 \pm 0.005$ \\
\hline $6^{\mathrm{Eg}}$ & 14.5 & 352 & 667 & $505(100), 301(42)$ & Quercetin- $O$-acetylglucoside- $O$-glucoside & $\operatorname{Tr}$ \\
\hline $7^{\mathrm{Eg}}$ & 15.8 & 348 & 651 & $489(38), 447(55), 285(56)$ & Kaempferol-O-acetylglucoside- $O$-glucoside & $5.01 \pm 0.05$ \\
\hline $8^{\mathrm{Eg}}$ & 18.8 & 336 & 739 & $593(77), 431(10), 285(27)$ & Kaempferol-O-rhamnoside- $O$-rutinoside & $0.67 \pm 0.04$ \\
\hline $9^{\mathrm{Eg}}$ & 19.2 & 348 & 593 & $447(41), 431$ (51), 285 (58) & Kaempferol-O-glucoside- $O$-rhamnoside & $1.86 \pm 0.02$ \\
\hline $10^{\mathrm{Eg}}$ & 21.2 & 358 & 463 & $301(100)$ & Quercetin-3-O-glucoside & $\operatorname{Tr}$ \\
\hline $11^{\mathrm{Eg}}$ & 23.1 & 348 & 593 & $285(100)$ & Kaempferol-3-O-rutinoside & $1.95 \pm 0.03$ \\
\hline $12^{\mathrm{Eg}}$ & 23.8 & 350 & 635 & 489 (13), 431 (55), 285 (31) & Kaempferol-O-acetylglucoside- $O$-rhamnoside & $1.77 \pm 0.06$ \\
\hline $13^{\mathrm{Eg}}$ & 24.7 & 350 & 447 & $285(100)$ & Kaempferol-3-O-glucoside & $1.81 \pm 0.03$ \\
\hline \multirow[t]{4}{*}{$14^{\mathrm{Eg}}$} & 30.1 & 350 & 489 & $285(100)$ & Kaempferol-O-acetylglucoside & $0.88 \pm 0.03$ \\
\hline & & & & & Total phenolic acids & $4.98 \pm 0.03$ \\
\hline & & & & & Total flavonoids & $21.7 \pm 0.4$ \\
\hline & & & & & Total phenolic compounds & $26.6 \pm 0.3$ \\
\hline
\end{tabular}

Tr - traces. 
Table 2 Phenolic compounds' identification and quantification in the Tilia platyphyllos extract

\begin{tabular}{|c|c|c|c|c|c|c|}
\hline Compound & $\begin{array}{l}R_{\mathrm{t}} \\
(\mathrm{min})\end{array}$ & $\begin{array}{l}\lambda_{\max } \\
(\mathrm{nm})\end{array}$ & $\begin{array}{l}\text { Molecular ion } \\
{[\mathrm{M}-\mathrm{H}]^{-}(\mathrm{m} / \mathrm{z})}\end{array}$ & $\mathrm{MS}^{2}(m / z)$ & Tentative identification & $\begin{array}{l}\text { Quantification } \\
\left(\mathrm{mg} \mathrm{g}^{-1} \text { extract }\right)\end{array}$ \\
\hline $1^{\mathrm{Tp}}$ & 5.1 & 328 & 353 & 191 (100), 179 (44), $161(5), 135$ (38) & 3-O-Caffeoylquinic acid & $0.64 \pm 0.03$ \\
\hline $2^{\mathrm{Tp}}$ & 6.1 & $\begin{array}{l}260 \\
294 \mathrm{sh}\end{array}$ & 153 & $109(100)$ & Protocatechuic acid & $6.3 \pm 0.1$ \\
\hline $3^{\mathrm{Tp}}$ & 7.2 & 280 & 289 & 245 (12), 203 (20), 187 (10), $161(5), 137$ (5) & $(+)$-Catechin & $1.6 \pm 0.1$ \\
\hline $4^{\mathrm{Tp}}$ & 8.1 & 328 & 353 & $191(100), 179(5), 135(6)$ & 5-O-Caffeoylquinic acid & $4.7 \pm 0.1$ \\
\hline $5^{\mathrm{Tp}}$ & 9.0 & 280 & 577 & $\begin{array}{l}451(26), 425(37), 407 \text { (100), } 289(69) \\
287(7)\end{array}$ & Procyanidin dimer B2 & $4.2 \pm 0.1$ \\
\hline $6^{\mathrm{Tp}}$ & 11.1 & 280 & 289 & 245 (8), 203 (22), 187 (11), 161 (7), 137 (7) & (-)-Epicatechin & $6.41 \pm 0.01$ \\
\hline $7^{\mathrm{Tp}}$ & 13.1 & 280 & 865 & $\begin{array}{l}739(10), 713(18), 577(34), 575(21) \\
425(14), 407(25), 289(15), 287(20)\end{array}$ & Procyanidin trimer & $2.54 \pm 0.02$ \\
\hline $8^{\mathrm{Tp}}$ & 14.2 & 280 & 1153 & $\begin{array}{l}865(6), 863(17), 577(61), 575(17), \\
289(42), 287(10)\end{array}$ & Procyanidin tetramer & $3.9 \pm 0.1$ \\
\hline $9^{\mathrm{Tp}}$ & 15.5 & 280 & 1441 & $1153(9), 865$ (9), 577 (31), $289(96), 287$ (30) & Procyanidin pentamer & $2.4 \pm 0.2$ \\
\hline $10^{\mathrm{Tp}}$ & 16.3 & 356 & 609 & $463(9), 447(27), 301(12)$ & $\begin{array}{l}\text { Quercetin- } O \text {-hexoside- } O \text { - } \\
\text { deoxyhexoside }\end{array}$ & $0.13 \pm 0.01$ \\
\hline $11^{\mathrm{Tp}}$ & 16.7 & 356 & 609 & $463(20), 447$ (45), $301(31)$ & $\begin{array}{l}\text { Quercetin-3-O-glucoside-7-O- } \\
\text { rhamnoside }\end{array}$ & $1.4 \pm 0.1$ \\
\hline $12^{\mathrm{Tp}}$ & 17.8 & $\begin{array}{l}286, \\
336 \text { sh }\end{array}$ & 449 & $287(100)$ & Eriodictyol-O-hexoside & $1.3 \pm 0.1$ \\
\hline $13^{\mathrm{Tp}}$ & 18.3 & 358 & 579 & 447 (64), 433 (10), 301 (33) & $\begin{array}{l}\text { Quercetin-O-pentoside-O- } \\
\text { rhamnoside }\end{array}$ & $0.65 \pm 0.05$ \\
\hline $14^{\mathrm{Tp}}$ & 19.2 & 348 & 593 & $447(41), 431(54), 285(33)$ & $\begin{array}{l}\text { Kaempferol-3-O-glucoside-7- } \\
O \text {-rhamnoside }\end{array}$ & $1.52 \pm 0.02$ \\
\hline $15^{\mathrm{Tp}}$ & 19.5 & 356 & 609 & $301(100)$ & $\begin{array}{l}\text { Quercetin-3-O-rutinoside } \\
\text { (rutin) }\end{array}$ & $0.96 \pm 0.02$ \\
\hline $16^{\mathrm{Tp}}$ & 19.8 & 352 & 593 & 446 (53), 301 (39) & $\begin{array}{l}\text { Quercetin-3,7-di-O- } \\
\text { rhamnoside }\end{array}$ & $1.1 \pm 0.1$ \\
\hline $17^{\mathrm{Tp}}$ & 20.2 & 350 & 477 & $301(100)$ & Quercetin-3-O-glucuronide & $0.21 \pm 0.05$ \\
\hline $18^{\mathrm{Tp}}$ & 20.5 & 348 & 563 & $431(66), 417$ (26), 285 (39) & $\begin{array}{l}\text { Kaempferol- } O \text {-pentoside- } O \text { - } \\
\text { deoxyhexoside }\end{array}$ & $0.76 \pm 0.05$ \\
\hline $19^{\mathrm{Tp}}$ & 20.6 & 358 & 463 & $301(100)$ & $\begin{array}{l}\text { Quercetin-3-O-galactoside } \\
\text { (hyperoside) }\end{array}$ & $1.01 \pm 0.02$ \\
\hline $20^{\mathrm{Tp}}$ & 21.1 & 356 & 463 & $301(100)$ & $\begin{array}{l}\text { Quercetin-3-O-glucoside } \\
\text { (isoquercitrin) }\end{array}$ & $6.36 \pm 0.04$ \\
\hline $21^{\mathrm{Tp}}$ & 22.5 & 360 & 505 & 463 (12), 301 (100) & Quercetin-3-O-acetylglucoside & $0.090 \pm 0.004$ \\
\hline $22^{\mathrm{Tp}}$ & 22.9 & $\begin{array}{l}284, \\
334 \text { sh }\end{array}$ & 433 & $271(100)$ & Naringenin- $O$-hexoside & $0.90 \pm 0.05$ \\
\hline $23^{\mathrm{Tp}}$ & 23.1 & 346 & 593 & $285(100)$ & Kaempferol-3-O-rutinoside & $1.03 \pm 0.01$ \\
\hline $24^{\mathrm{Tp}}$ & 23.4 & 346 & 577 & $431(72), 285(78)$ & $\begin{array}{l}\text { Kaempferol-3,7-di-O- } \\
\text { rhamnoside }\end{array}$ & $1.15 \pm 0.03$ \\
\hline $25^{\mathrm{Tp}}$ & 23.7 & 356 & 433 & $301(100)$ & Quercetin-O-pentoside & $1.8 \pm 0.1$ \\
\hline $26^{\mathrm{Tp}}$ & 24.8 & 350 & 447 & $301(100)$ & $\begin{array}{l}\text { Quercetin-3-O-rhamnoside } \\
\text { (quercitrin) }\end{array}$ & $3.5 \pm 0.1$ \\
\hline $27^{\mathrm{Tp}}$ & 26.1 & 348 & 447 & $285(100)$ & Kaempferol-O-hexoside & $0.55 \pm 0.03$ \\
\hline $28^{\mathrm{Tp}}$ & 26.8 & 352 & 417 & $285(100)$ & Kaempferol-O-pentoside & $0.38 \pm 0.03$ \\
\hline $29^{\mathrm{Tp}}$ & 29.2 & 346 & 431 & $285(100)$ & Kaempferol-O-rhamnoside & $0.39 \pm 0.03$ \\
\hline $30^{\mathrm{Tp}}$ & 30.7 & 350 & 431 & $285(100)$ & Kaempferol-O-rhamnoside & $0.45 \pm 0.04$ \\
\hline $31^{\mathrm{Tp}}$ & 32.5 & 332 & 283 & $269(100)$ & Methyl apigenin & $2.07 \pm 0.01$ \\
\hline $32^{\mathrm{Tp}}$ & 35.0 & 316 & 593 & 447 (10), 285 (79) & trans-Tiliroside & $1.38 \pm 0.03$ \\
\hline \multirow[t]{4}{*}{$33^{\mathrm{Tp}}$} & 35.3 & 326 & 593 & $447(10), 285$ (79) & cis-Tiliroside & $0.27 \pm 0.03$ \\
\hline & & & & & Total phenolic acids & $11.65 \pm 0.05$ \\
\hline & & & & & Total flavonoids & $50.4 \pm 0.4$ \\
\hline & & & & & Total phenolic compounds & $62.0 \pm 0.4$ \\
\hline
\end{tabular}

quercetin-3-O-glucoside have also been found in commercial samples of T. platyphyllos from Syria and Tuscany. ${ }^{21}$

Besides the already mentioned phenolic acid derivatives, 3-O-caffeoylquinic acid (peak $11^{\mathrm{TP}}$ ) was also tentatively identified based on its $\mathrm{MS}^{2}$ fragmentation pattern, according to the systematic keys provided by Clifford et al. ${ }^{26}$ for the identification of chlorogenic acids. Phenolic acid derivatives represent $19 \%$ of the total phenolic composition of T. platyphyllos.

The sample of T. platyphyllos presented four compounds, $5^{\mathrm{TP}}\left([\mathrm{M}-\mathrm{H}]^{-}\right.$at $\mathrm{m} / z$ 577), $7^{\mathrm{TP}}\left([\mathrm{M}-\mathrm{H}]^{-}\right.$at $\mathrm{m} / z$ 865),
$8^{\mathrm{TP}}\left([\mathrm{M}-\mathrm{H}]^{-}\right.$at $\left.m / z 1153\right)$ and $9^{\mathrm{TP}}\left([\mathrm{M}-\mathrm{H}]^{-}\right.$at $\left.m / z 1441\right)$, with characteristics of proanthocyanidins (UV spectra with $\lambda_{\max }$ $280 \mathrm{~nm}$ ), being assigned as a procyanidin dimer, trimer, tetramer and pentamer, respectively. A comparison with our library database allowed identifying peak $5^{\mathrm{TP}}$ as procyanidin dimer B2, also reported by Negri et al. ${ }^{27}$ in hydroethanolic extracts from leaves of Tilia cordata. These compounds have been reported as major compounds in samples of $T$. platyphyllos by Karioti et $a l .{ }^{21}$ In the present study, these compounds were also present in high amounts, representing $34 \%$ of the total 

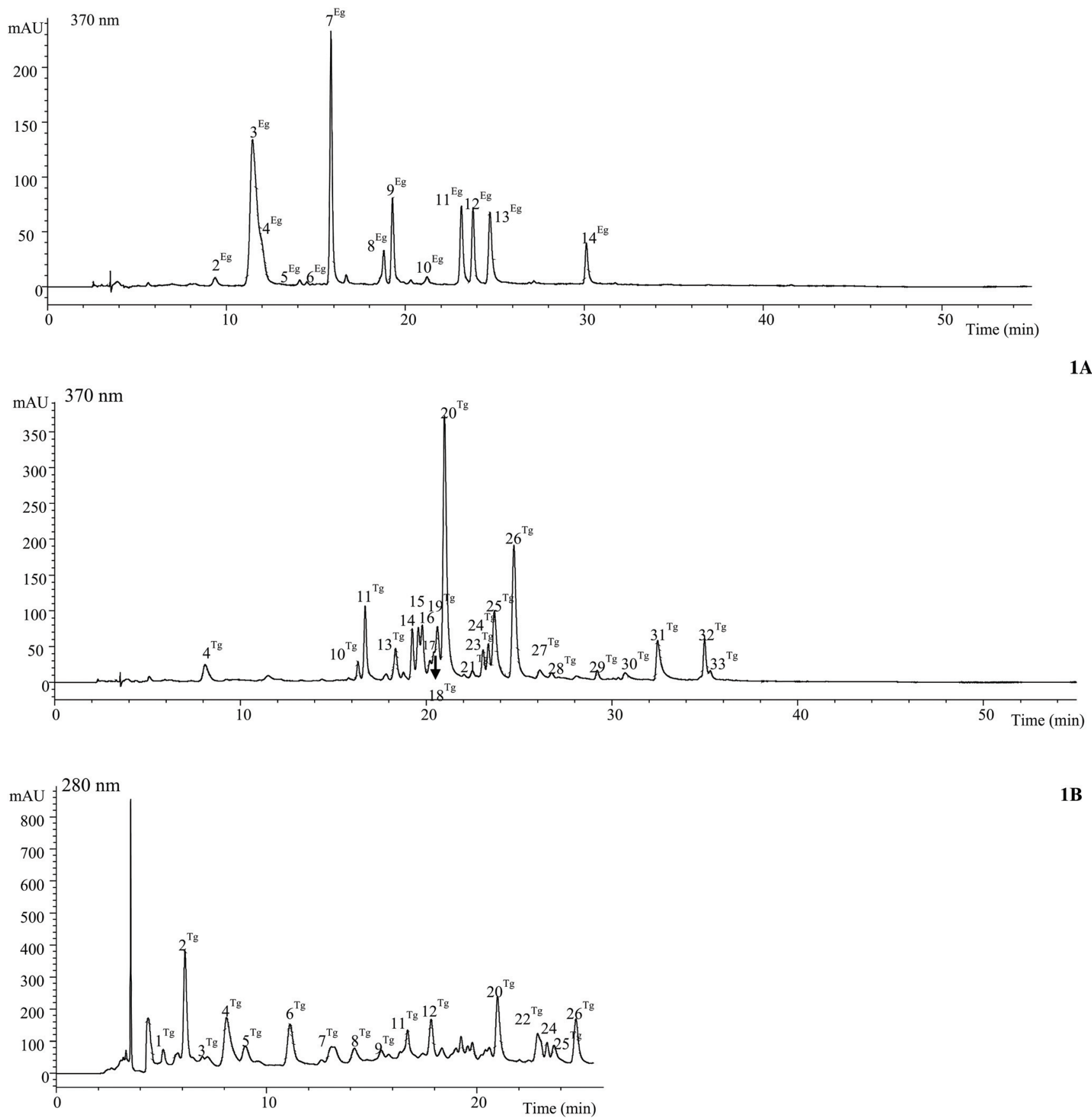

Fig. 1 Phenolic compound profiles of E. giganteum recorded at $370 \mathrm{~nm}$ (A) and T. platyphyllos recorded at 370 and $280 \mathrm{~nm}$ (B). The numbering corresponds to the peak number presented in Tables 1 and 2.

phenolic content, including (+)-catechin, (-)-epicatechin being the most abundant compound found $\left(6.4 \mathrm{mg} \mathrm{g}^{-1}\right.$ extract).

Peaks $10^{\mathrm{Tp}}, 11^{\mathrm{Tp}}, 13^{\mathrm{Tp}}, 16^{\mathrm{Tp}}, 19^{\mathrm{Tp}}, 21^{\mathrm{Tp}}, 25$ and $26^{\mathrm{Tp}}$ were identified as quercetin derivatives owing to the product ion observed at $m / z \quad 301$ and their UV spectra ( $\lambda_{\max }$ around 350-358 nm). Peaks $10^{\mathrm{TP}}$ and $11^{\mathrm{Tp}}$ both presented the same pseudomolecular ion $\left([\mathrm{M}-\mathrm{H}]^{-}\right.$at $\left.m / z 609\right)$ as peak $15^{\mathrm{Tp}}$ (quercetin-3-O-rutinoside), but a different fragmentation pattern as in those compounds product ions from the alternative loss of hexosyl $(\mathrm{m} / \mathrm{z}$ at $447 ;-162 \mathrm{u})$ and deoxyhexosyl $(\mathrm{m} / \mathrm{z}$ at 463 ; $-146 \mathrm{u}$ ) were observed, indicating their location at different positions of the aglycone. Compounds with similar features have been reported in T. platyphyllos ${ }^{21}$ and $T$. cordata $^{27}$ and identified as quercetin-3-O-glucoside-7-O-rhamnoside, an identity that was tentatively assumed for peak $11^{\mathrm{Tp}}$ in our sample owing to its greater concentration, whereas peak $10^{\mathrm{Tp}}$ remained just assigned as quercetin- $O$-hexoside- $O$-deoxyhexoside. Peak $16^{\mathrm{Tp}}$ showed a pseudomolecular ion $[\mathrm{M}-\mathrm{H}]^{-}$at $\mathrm{m} / \mathrm{z}$ 
593, yielding two fragment ions from the consecutive loss of two deoxyhexosyl residues; it was tentatively identified as quercetin3,7-di-O-rhamnoside, a compound commonly reported in Tilia species $^{27-30}$ including T. platyphyllos, ${ }^{21}$ whereas peak $13^{\mathrm{Tp}}$ $\left([\mathrm{M}-\mathrm{H}]^{-}\right.$at $\left.m / z 579\right)$ was tentatively assigned as quercetin- $O$ pentoside- $O$-rhamnoside, as reported in $T$. cordata by Negri et al. ${ }^{27}$ Compounds $19^{\mathrm{Tp}}\left([\mathrm{M}-\mathrm{H}]^{-}\right.$at $\left.m / z 463\right), 25^{\mathrm{Tp}}\left([\mathrm{M}-\mathrm{H}]^{-}\right.$ at $m / z 433)$ and $26^{\mathrm{Tp}}\left([\mathrm{M}-\mathrm{H}]^{-}\right.$at $\left.m / z 447\right)$, presented $\mathrm{MS}^{2}$ fragments corresponding to distinct losses of hexosyl (-162 u), pentosyl (-132 u) and deoxyhexosyl (-146 u) moieties, and an elution order coherent with the type of sugar substituents, according to their expected polarity. Although the position and nature of the sugar moieties could not be identified, an identity as quercetin-3-O-rhamnoside (quercitrin) was assumed for compound $26^{\mathrm{Tp}}$, previously described in T. platyphyllos ${ }^{21}$ and other Tilia species. ${ }^{27,29,30}$ Similarly, peak $19^{\mathrm{TP}}$ was tentatively assigned as quercetin-3-O-galactoside (hyperoside) owing to its previous identification in Tilia tomentosa ${ }^{30}$ and T. cordata $^{31}$ that identity would also be supported by its close elution to quercetin-3-O-glucoside (peak $20^{\mathrm{Tp}}$ ), since galactosides typically elute before the equivalent glucosides in RP-HPLC. ${ }^{32}$ Peak $21^{\mathrm{TP}}\left([\mathrm{M}-\mathrm{H}]^{-}\right.$at $\left.m / z 505\right)$, with a molecular weight $42 \mathrm{u}$ (acetyl residue) higher than compounds $19^{\mathrm{Tp}}$ and $20^{\mathrm{Tp}}$ was tentatively identified as quercetin-3-O-acetylglucoside, assuming that it could derive from the more abundant precursor.

Peaks $14^{\mathrm{Tp}}, 18^{\mathrm{Tp}}, 24^{\mathrm{Tp}}, 27^{\mathrm{Tp}}, 28^{\mathrm{Tp}}, 29^{\mathrm{Tp}}, 30^{\mathrm{Tp}}, 32^{\mathrm{Tp}}$ and $33^{\mathrm{Tp}}$ were identified as kaempferol glycosides based on their UV spectra ( $\lambda_{\max }$ around $348 \mathrm{~nm}$ ) and due to the production of an $\mathrm{MS}^{2}$ fragment ion at $\mathrm{m} / z 285$. Tentative identities of these compounds were assigned based on their pseudomolecular ions using a similar reasoning as for quercetin derivatives. Thus, peaks $14^{\mathrm{Tp}}\left([\mathrm{M}-\mathrm{H}]^{-}\right.$at $\left.m / z 593\right)$ and $24^{\mathrm{Tp}}\left([\mathrm{M}-\mathrm{H}]^{-}\right.$at $m / z$ 577 ) should correspond to kaempferol-3-O-glucoside-7-Orhamnoside and kaempferol-3,7-O-dirhamnoside, respectively, compounds that have been reported in T. platyphyllos ${ }^{21}$ and other Tilia species. ${ }^{27-30}$ Peaks $32^{\mathrm{TP}}$ and $33^{\mathrm{TP}}$ possessed the same pseudomolecular ion $\left([\mathrm{M}-\mathrm{H}]^{-}\right.$at $\left.m / z 593\right)$ as peaks $14^{\mathrm{Tp}}$ and $24^{\mathrm{Tp}}$ (kaempferol-3-O-rutinoside), but eluted at a longer retention time. The presence of tiliroside (kaempferol-3-O(6-p-coumaroyl)-glucoside; 6"-O-(4-hydroxycinnamoyl)-astragalin) has been consistently reported in different species of Tilia, ${ }^{27,29-31,33}$ including T. platyphyllos. ${ }^{21}$ The observation in their fragmentation of a product ion at $\mathrm{m} / \mathrm{z} 447$, from the loss of $p$-coumaroyl residue (146 u) would also be coherent with that identity, as well as with the late elution, since the presence of the hydroxycinnamoyl residue implies a decrease in polarity. The detection of two peaks could be explained by the existence of trans- and cis-isomers of the p-coumaric acid, as also noted by Karioti et al. ${ }^{21}$ The most abundant peak $32^{\mathrm{TP}}$ can be assigned as trans-tiliroside taking into account that the form trans is the usual one for $p$-coumaric acid. Peak $27^{\mathrm{Tp}}$ $\left([\mathrm{M}-\mathrm{H}]^{-}\right.$at $\left.m / z 447\right)$ presented the same pseudomolecular ion as kaempferol-3-O-glucoside (astragalin), a compound that was commonly detected in Tilia spp., ${ }^{21,27,29,31}$ but it eluted at a different retention time, as compared with a commercial standard; thus, the detected compound was just assigned as
kaempferol-O-hexoside. Peaks $29^{\mathrm{Tp}}$ and $30^{\mathrm{Tp}}$, with the same pseudomolecular ion $\left([\mathrm{M}-\mathrm{H}]^{-}\right.$at $\left.m / z 431\right)$ are coherent with kaempferol bearing it as a deoxyhexosyl moiety. Two undefined kaempferol rhamnosides were found in T. tomentosa by Ieri et al., ${ }^{30}$ while the presence of kaempferol-3-O-rhamnoside was reported in different Tilia spp. ${ }^{21,27,29}$ that could correspond to one of the compounds detected in our study. However, since no sufficient information to assign that identity to any of the two detected peaks could be obtained, both compounds remain identified as kaempferol-O-rhamnoside. The remaining two kaempferol derivatives, i.e., peaks $18^{\mathrm{TP}}$ and $28^{\mathrm{Tp}}$, were respectively assigned as kaempferol- $O$-pentoside- $O$ hexoside and kaempferol-O-pentoside, respectively, based on their mass spectral characteristics. As far as we are aware, none of these compounds has been previously reported in Tilia spp. Flavonols accounted for $41 \%$ of the content of total phenolic compounds, quercetin-3-O-glucoside being the main molecule of this group.

Compounds $12^{\mathrm{Tp}}\left([\mathrm{M}-\mathrm{H}]^{-}\right.$at $\left.m / z 449\right)$ and $22^{\mathrm{Tp}}\left([\mathrm{M}-\mathrm{H}]^{-}\right.$ at $m / z 433)$ presented UV spectra characteristics of flavanones and $\mathrm{MS}^{2}$ fragments at $m / z 287$ (eriodictyol) and at $m / z 271$ (naringenin), respectively, indicating the loss of an hexosyl residue $(162 \mathrm{u})$, so that they were tentatively assigned as eriodictyol$O$-hexoside and naringenin- $O$-hexoside. Finally, peak $31^{\mathrm{Tp}}$ showed a pseudomolecular ion $[\mathrm{M}-\mathrm{H}]^{-}$at $\mathrm{m} / \mathrm{z} 283$ that released an $\mathrm{MS}^{2}$ fragment at $m / z 269$ (apigenin, $[\mathrm{M}-\mathrm{H}-14]^{-}$) indicating the loss of a methyl group; this observation together with its UV spectrum, characteristic of a flavone, suggested that it might correspond to a methyl apigenin. To the best of our knowledge, neither flavanones nor flavones have been described in T. platyphyllos.

Equisetum giganteum mainly presented kaempferol derivatives, accounting for $81 \%$ of the total phenolic content. Thus, in addition to peaks $11^{\mathrm{Eg}}$ and $13^{\mathrm{Eg}}$, positively identified as kaempferol-3-O-rutinoside and kaempferol-3-O-glucoside, peaks $3^{\mathrm{Eg}}, 5^{\mathrm{Eg}}, 7^{\mathrm{Eg}}, 8^{\mathrm{Eg}}, 12^{\mathrm{Eg}}$ and $14^{\mathrm{Eg}}$ were also assigned as kaempferol-derived compounds based on their UV spectra ( $\lambda_{\max }$ around $348 \mathrm{~nm}$ ) and the observation of a product ion at $\mathrm{m} / \mathrm{z} 285$; tentative identities were attributed from their molecular weight and fragmentation patterns.

Compound $3^{\mathrm{Eg}}\left([\mathrm{M}-\mathrm{H}]^{-}\right.$at $m / z$ 755) $\mathrm{MS}^{2}$ fragments revealed the alternative loss of hexosyl $(\mathrm{m} / \mathrm{z}$ at $593 ;-162 \mathrm{u})$ and deoxyhexosyl-hexoside $(\mathrm{m} / \mathrm{z}$ at $447 ;-308 \mathrm{u})$ residues, indicating the location of each residue on different positions of the aglycone. The positive identification of kaempferol-3-O-rutinoside and kaempferol-3-O-glucoside may suggest the presence of glucosyl and rutinosyl as sugar substituents, so that the compound was tentatively assigned as kaempferol-O-glucoside- $O$ rutinoside. This molecule was the most abundant phenolic compound (7.6 $\mathrm{mg} \mathrm{g}^{-1}$ extract) present in this sample. Similarly, compound $8^{\mathrm{Eg}}\left([\mathrm{M}-\mathrm{H}]^{-}\right.$at $\left.m / z 739\right)$ was assigned as kaempferol-O-rhamnoside- $O$-rutinoside.

Peaks $5^{\mathrm{Eg}}$ and $7^{\mathrm{Eg}}$, with the same $[\mathrm{M}-\mathrm{H}]^{-}$at $\mathrm{m} / z 651$ and similar fragmentation pattern, with the production of fragment ions at $\mathrm{m} / \mathrm{z} 489$ and 447 , from the alternative loss of hexosyl $(-162 \mathrm{u})$ and acetylhexosyl residues $(-204 \mathrm{u})$, were assigned as 
kaempferol-O-acetylglucoside-O-glucoside. The observation of two compounds might be explained by different substitution positions of either each of the two residues on the aglycone or the acetyl moiety on the glucose. Compound $9^{\mathrm{Eg}}\left([\mathrm{M}-\mathrm{H}]^{-}\right.$at $\mathrm{m} / \mathrm{z} 593$ ) showed the same pseudomolecular ion as kaempferol3-O-rutinoside but a different fragmentation pattern. The observation of product ions at $m / z 447(-146 \mathrm{u})$ and $431(-162 \mathrm{u})$ revealed the alternative loss of deoxyhexosyl and hexosyl residues, allowing its identification as kaempferol-O-glucoside- $O$ rhamnoside, a compound that was reported in extracts of the aerial parts of Equisetum telmateia. ${ }^{34}$ Peak $12^{\text {Eg }}\left([\mathrm{M}-\mathrm{H}]^{-}\right.$at $\mathrm{m} / \mathrm{z} 635$ ) presented a molecular weight $42 \mathrm{u}$ greater than peak $9^{\mathrm{Eg}}$ and $\mathrm{MS}^{2}$ fragment ions at $m / z 489(-146 \mathrm{u}$, loss of a rhamnosyl residue) and $431(-204 \mathrm{u}$, loss of an acetylhexosyl residue), being identified as kaempferol- $O$-acetylglucoside- $O$ rhamnoside. Peak $14^{\mathrm{Eg}}$, with an $[\mathrm{M}-\mathrm{H}]^{-}$at $m / z 489$ yielding a unique fragment ion at $m / z 285$, from the loss of an acetylhexosyl residue, was assigned as kaempferol-3-O-acetylglucoside, a compound also reported by Correia et al. ${ }^{34}$ in E. telmateia.

Peaks $2^{\mathrm{Eg}}$ and $6^{\mathrm{Eg}}$ were assigned as quercetin derivatives based on their absorption spectra and the production of a fragment ion at $\mathrm{m} / \mathrm{z}$ 301. Based on their fragmentation features and following a similar reasoning as for the previous kaempferol derivatives, they were assigned as quercetin-di-O-glucoside and quercetin- $O$-acetylglucoside- $O$-glucoside, respectively. Peak $2^{\mathrm{Eg}}$ was tentatively identified as quercetin-3,7-di-O-glucoside, owing to the previous description of such a compound in E. giganteum by Francescato et al. ${ }^{15}$ and Alavarce et al. ${ }^{16}$

Finally, peak $1^{\mathrm{Eg}}$ was identified as a caffeic acid hexoside according to its UV and mass characteristics.

With the exception of quercetin-3-O-glucoside and kaempferol-3-O-rutinoside, reported in E. giganteum by Francescato et $a l .{ }^{15}$ and Alavarce et al. ${ }^{16}$ none of the compounds detected herein had been previously described in this species.

\subsection{Evaluation of the antioxidant activity}

The results of the antioxidant activity of both plant extracts are shown in Table 3. In general, T. platyphyllos evidenced a higher antioxidant potential than $E$. giganteum, both as a free radical scavenger, and also as a lipid peroxidation inhibitor, these results being clearly confirmed, respectively, by the lowest RSA, RP, CBI and LPI $\mathrm{EC}_{50}$ values. Not least interesting to highlight is that this biological activity seems to be directly correlated with the relative abundance in phenolic compounds: for the plant extract with higher antioxidant effects, i.e. T. platyphyllos, a high concentration of phenolic compounds was found (Tables 1 and 2). Thus, it is feasible to infer that the most prominent antioxidant potential evidenced to T. platyphyllos, through all the in vitro assays performed, was mainly attributed to their richness in phenolic compounds, particularly flavonoids (quercetin and kaempferol derivatives), as shown in Table 2 .

Majer et al. ${ }^{20}$ aiming to assess the in vitro antioxidant potential (singlet oxygen scavenging) of T. platyphyllos leaf flavonoids, concluded that sun leaves possess a higher myricetin content than shade leaves, and that quercetin and myricetin derivatives,
Table 3 Antioxidant, antitumor and anti-inflammatory activities of the studied plant extracts

Student's $t$-test

E. giganteum T. platyphyllos $p$-Value

\begin{tabular}{|c|c|c|c|}
\hline \multicolumn{4}{|c|}{ Antioxidant activity $\left(\mathrm{EC}_{50}\right.$ values, $\left.\mu \mathrm{g} \mathrm{mL}^{-1}\right)$} \\
\hline $\begin{array}{l}\text { DPPH scavenging } \\
\text { activity (RSA) }\end{array}$ & $123 \pm 5$ & $105 \pm 1$ & $<0.001$ \\
\hline Reducing power (RP) & $136 \pm 1$ & $123 \pm 7$ & $<0.001$ \\
\hline $\begin{array}{l}\beta \text {-Carotene bleaching } \\
\text { inhibition (CBI) }\end{array}$ & $202 \pm 3$ & $167 \pm 2$ & $<0.001$ \\
\hline $\begin{array}{l}\text { TBARS inhibition } \\
\text { (LPI) }\end{array}$ & $57 \pm 1$ & $56 \pm 1$ & 0.024 \\
\hline \multicolumn{4}{|c|}{ Antitumor activity $\left(\mathrm{GI}_{50}\right.$ values, $\left.\mu \mathrm{g} \mathrm{mL}^{-1}\right)$} \\
\hline $\begin{array}{l}\text { MCF-7 (breast } \\
\text { carcinoma) }\end{array}$ & $250 \pm 15$ & $224 \pm 19$ & 0.058 \\
\hline $\begin{array}{l}\text { NCI-H460 (non-small } \\
\text { cell lung cancer) }\end{array}$ & $258 \pm 13$ & $247 \pm 22$ & 0.380 \\
\hline $\begin{array}{l}\text { HeLa (cervical } \\
\text { carcinoma) }\end{array}$ & $268 \pm 16$ & $195 \pm 15$ & 0.001 \\
\hline HepG2 & $239 \pm 18$ & $173 \pm 13$ & 0.002 \\
\hline
\end{tabular}

(hepatocellular

carcinoma)

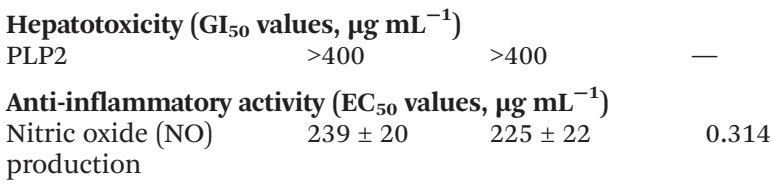

The antioxidant activity was expressed as $\mathbf{E C}_{50}$ values, which means that higher values correspond to a lower reducing power or antioxidant potential. $\mathrm{EC}_{50}$ : extract concentration corresponding to $50 \%$ of antioxidant activity or 0.5 of absorbance in the reducing power assay. Trolox $\mathrm{EC}_{50}$ values: $41 \mu \mathrm{g} \mathrm{mL} \mathrm{m}^{-1}$ (reducing power), $42 \mu \mathrm{g} \mathrm{mL} \mathrm{m}^{-1}$ (DPPH scavenging activity), $18 \mu \mathrm{g} \mathrm{mL} \mathrm{m}^{-1}$ ( $\beta$-carotene bleaching inhibition) and $23 \mu \mathrm{g} \mathrm{mL} \mathrm{m}^{-1}$ (TBARS inhibition). Results of the anti-inflammatory activity are expressed in $\mathrm{EC}_{50}$ values: sample concentration providing $50 \%$ of inhibition of nitric oxide (NO) production. Dexamethasone $\mathrm{EC}_{50}$ value: $16 \pm 2 \mu \mathrm{g} \mathrm{mL}^{-1}$. Cytotoxicity results are expressed in $\mathrm{GI}_{50}$ values corresponding to the sample concentration achieving $50 \%$ of growth inhibition in human tumor cell lines or in liver primary culture PLP2. Ellipticine GI $\mathrm{G}_{50}$ values: $1.2 \mu \mathrm{g} \mathrm{mL}^{-1}$ (MCF-7), $1.0 \mu \mathrm{g} \mathrm{mL}^{-1}$ (NCI-H460), $0.91 \mu \mathrm{g} \mathrm{mL}^{-1}$ (HeLa), $1.1 \mu \mathrm{g} \mathrm{mL}^{-1}$ (HepG2) and 2.3 (PLP2).

mainly occurring as rhamnosides, exerted much higher singlet oxygen scavenger effects than kaempferol glycosides. In fact, a higher content in quercetin derivatives was observed in T. platyphyllos, to the detriment of kaempferol derivatives; furthermore, in E. giganteum only vestigial amounts of quercetin were determined, kaempferol being the most abundant. On the other hand, Yayalaci et al. ${ }^{19}$ evaluating the in vivo antioxidant potential of T. platyphyllos flowers against ethanol-induced oxidative stress, concluded that linden flowers were able to not only prevent oxidative damage in the studied tissues, but also to inhibit the production of ethanol-induced free radicals in rats. Thus, and considering the obtained results, it is feasible to suppose that the antioxidant activity of T. platyphyllos is directly correlated with the relative abundance in phenolic compounds, mainly quercetin derivatives.

\subsection{Evaluation of the anti-inflammatory activity}

The anti-inflammatory potential of both plant extracts was evaluated through the measurement of nitric oxide (NO) 
production, and the obtained results are presented in Table 3. Once again, the phenolic composition seems to exert a determinant role in the anti-inflammatory potential of plant extracts: T. platyphyllos appears to be more effective than E. giganteum because a lower concentration of the plant extract was necessary to achieve $50 \%$ of inhibition of NO production, than using E. giganteum. As previously mentioned, only Farinon et al. ${ }^{17}$ assessed the in vivo anti-inflammatory potential of E. giganteum; otherwise, to the author's best knowledge, no studies have been previously reported on the anti-inflammatory potential of T. platyphyllos. However, the in vitro antiinflammatory potential of other phenolic matrices has been also previously assessed by other authors. ${ }^{35,36}$ Chen and Kang $^{35}$ by assessing the in vitro anti-inflammatory potential of a methanolic extract obtained from Capsicum annuum L. (red pepper) stalk extracts, observed that a concentration of 100 $\mu \mathrm{g} \mathrm{mL}^{-1}$ was able to inhibit $53.5 \%$ of NO production. On the other hand, Silva et al. $^{36}$ aiming to assess the in vitro antiinflammatory potential of three different phenolic extracts (two aqueous and one methanol extract) obtained from Gomphrena globosa L. inflorescences reported that aqueous extracts were able to inhibit by $50 \%$ the NO production at $472.3 \pm 31.3 \mu \mathrm{g} \mathrm{mL}^{-1}$ (decoction) and $1260 \pm 50.5 \mu \mathrm{g} \mathrm{mL}^{-1}$ (infusion), while for methanolic extracts, a concentration of $1166.0 \pm 16.7 \mu \mathrm{g} \mathrm{mL}{ }^{-1}$ was necessary.

Thus, considering the results obtained in the present study, despite $E$. giganteum also acting as an anti-inflammatory agent, the effect of T. platyphyllos was higher; therefore, it may be considered a rich source of phenolic compounds with antiinflammatory potential. In fact, a concentration of $225 \pm 22$ $\mu \mathrm{g} \mathrm{mL}^{-1}$ of $T$. platyphyllos hydroethanolic extract was effective at inhibiting $50 \%$ of NO production, while for E. giganteum it was necessary to use $239 \pm 20 \mu \mathrm{g} \mathrm{mL}{ }^{-1}$.

\subsection{Evaluation of the cytotoxic activity}

The cytotoxicity of both plant extracts was evaluated in nontumor porcine liver cells (PLP2) and also in tumor cell lines (breast carcinoma - MCF-7, non-small cell lung carcinoma NCI-H460, cervical carcinoma - HeLa and hepatocellular carcinoma - HepG2), and the results are given in Table 3. Neither E. giganteum nor T. platyphyllos extracts showed toxicity in nontumor liver cells (PLP2). On the other hand, a relatively low concentration of the extracts was necessary to cause $50 \%$ of the growth inhibition in human tumor cell lines. Once again, T. platyphyllos was revealed to be the more effective than E. giganteum, for all the tested tumor cell lines. Cytotoxic properties of the mentioned extracts were not previously mentioned in the literature.

Overall, the total phenolic compounds, phenolic acids and flavonoids were highly correlated with the antioxidant, antiinflammatory and antitumor activities of both samples, presenting high correlation factors, especially for the DPPH scavenging activity $\left(R^{2}=0.89\right)$, reducing power $\left(R^{2}=0.66\right), \beta$-carotene bleaching inhibition $\left(R^{2}=0.98\right)$, TBARS $\left(R^{2}=0.73\right)$, and for human tumor cell lines HeLa $\left(R^{2}=0.94\right)$, HepG2 $\left(R^{2}=0.93\right)$ and MCF-7 $\left(R^{2}=0.62\right)$, confirming the statements mentioned above.

\section{Conclusion}

Hydroethanolic extracts rich in phenolic compounds obtained from E. giganteum and T. platyphyllos seem to be very interesting matrices for a wide variety of conditions. The in vitro antioxidant, anti-inflammatory and antitumor effects were further investigated in this experiment, with promissory results being achieved. T. platyphyllos showed a higher biological potential than E. giganteum, its phenolic compound content and composition being directly correlated with the observed potentialities. In fact, in T. platyphyllos a higher content of total flavonoids (50.4 \pm $0.4 \mathrm{mg} \mathrm{g}^{-1}$ ) and phenolic acids (11.65 $\pm 0.05 \mathrm{mg} \mathrm{g}^{-1}$ ) was reached; however, despite the observed biochemical differences, E. giganteum also gave very positive results. Its content of total flavonoids and phenolic acids was significantly lower than that obtained for $T$. platyphyllos, $21.7 \pm 0.4 \mathrm{mg} \mathrm{g}^{-1}$ and $4.98 \pm$ $0.03 \mathrm{mg} \mathrm{g}^{-1}$, respectively, but the observed biological potential was also very interesting. While in T. platyphyllos the most abundant phenolic acid and flavonoid were, respectively, protocatechuic acid and (-)-epicatechin, followed by quercetin 3-O-glucoside, in $E$. giganteum the most abundant compounds were caffeic acid and kaempferol-O-glucoside- $O$-rutinoside, respectively.

Thus, in conclusion, despite the obtained results for both phenolic matrices, further studies are necessary to assess the in vivo efficacy, the involved mechanism of action and the related therapeutic doses of both plant extracts.

\section{Author contribution}

L. Achour and I. C. F. R. Ferreira conceived the study. I. Jabeur and N. Martins carried out the experiments. L. Barros gave support in the analysis of phenolic compounds. R. C. Calhelha and J. Vaz gave support for antitumor assays. L. Barros, I. C. F. R. Ferreira and C. Santos-Buelga performed data organization and analysis of the results. I. Jabeur, N. Martins and L. Barros wrote the manuscript. I. C. F. R. Ferreira, L. Achour and C. Santos-Buelga revised the manuscript.

\section{Conflict of interest}

The authors declare that there are no conflicts of interest.

\section{Acknowledgements}

The authors are grateful to Fundação para a Ciência e a Tecnologia (FCT, Portugal) for financial support to CIMO (Pest-OE/AGR/UI0690/2015) and to POCI-01-0145-FEDER006984 (LA LSRE-LCM) funded by ERDF through POCICOMPETE2020 and FCT. L. Barros and N. Martins thank FCT for their grants (SFRH/BPD/107855/2015 and SFRH/BD/87658/ 2012, respectively). The GIP-USAL is financially supported by the Spanish Government through the project AGL2015-64522C2-2-R. 


\section{References}

1 B. Holst and G. Williamson, Nutrients and phytochemicals: from bioavailability to bioefficacy beyond antioxidants, Curr. Opin. Biotechnol., 2008, 19(2), 73-82, DOI: 10.1016/j. copbio.2008.03.003.

2 J. C. Espín, M. T. García-Conesa and F. A. Tomás-Barberán, Nutraceuticals: facts and fiction, Phytochemistry, 2007, 68(22-24), 2986-3008, DOI: 10.1016/j.phytochem.2007.09.014.

3 S. Subash, M. M. Essa, S. Al-Adawi, et al., Neuroprotective effects of berry fruits on neurodegenerative diseases, Neural Regener. Res., 2014, 9(16), 1557, DOI: 10.4103/16735374.139483.

4 M. A. Soobrattee, V. S. Neergheen, A. Luximon-Ramma, O. I. Aruoma and T. Bahorun, Phenolics as potential antioxidant therapeutic agents: Mechanism and actions, Mutat. Res., Fundam. Mol. Mech. Mutagen., 2005, 579(1-2), 200213, DOI: 10.1016/j.mrfmmm.2005.03.023.

5 N. Martins, L. Barros, M. Henriques, S. Silva and I. C. F. R. Ferreira, Activity of phenolic compounds from plant origin against Candida species, Ind. Crops Prod., 2015, 74, 648-670, DOI: 10.1016/j.indcrop.2015.05.067.

6 C. Santos-Buelga, S. Gonzalez-Manzano, M. Dueñas and A. M. Gonzalez-Paramas, Extraction and isolation of phenolic compounds, Methods Mol. Biol., 2012, 864, 427464.

7 J. P. E. Spencer, K. Vafeiadou, R. J. Williams and D. Vauzour, Neuroinflammation: Modulation by flavonoids and mechanisms of action, Mol. Aspects Med., 2012, 33(1), 83-97, DOI: 10.1016/j.mam.2011.10.016.

8 F. Bourgaud, A. Gravot, S. Milesi and E. Gontier, Production of plant secondary metabolites: a historical perspective, Plant Sci., 2001, 161(5), 839-851, DOI: 10.1016/ S0168-9452(01)00490-3.

9 L. Rubió, M.-J. Motilva and M.-P. Romero, Recent advances in biologically active compounds in herbs and spices: a review of the most effective antioxidant and anti-inflammatory active principles, Crit. Rev. Food Sci. Nutr., 2013, 53(9), 943-953, DOI: 10.1080/10408398.2011.574802.

10 M. Viuda-Martos, Y. Ruiz-Navajas, J. Fernández-López and J. A. Pérez-Álvarez, Spices as Functional Foods, Crit. Rev. Food Sci. Nutr., 2010, 51(1), 13-28, DOI: 10.1080/ 10408390903044271.

11 A. Li, S. Li, Y. Zhang, X. Xu, Y. Chen and H. Li, Resources and biological activities of natural polyphenols, Nutrients, 2014, 6, 6020-6047, DOI: 10.3390/nu6126020.

12 M. S. Gião, C. I. Pereira, M. E. Pintado and F. X. Malcata, Effect of technological processing upon the antioxidant capacity of aromatic and medicinal plant infusions: From harvest to packaging, LWT-Food Sci. Technol., 2013, 50(1), 320-325, DOI: 10.1016/j.lwt.2012.05.007.

13 J. Cheel, L. Tůmová, C. Areche, et al., Variations in the chemical profile and biological activities of licorice (Glycyrrhiza glabra L.), as influenced by harvest times, Acta Physiol. Plant., 2012, 35(4), 1337-1349, DOI: 10.1007/ s11738-012-1174-9.
14 N. Martins, L. Barros, C. Santos-Buelga, M. Henriques, S. Silva and I. C. F. R. Ferreira, Evaluation of bioactive properties and phenolic compounds in different extracts prepared from Salvia officinalis L., Food Chem., 2014, 170, 378-385, DOI: 10.1016/j.foodchem.2014.08.096.

15 L. N. Francescato, S. L. Debenedetti, T. G. Schwanz, V. L. Bassani and A. T. Henriques, Identification of phenolic compounds in Equisetum giganteum by LC-ESI-MS/MS and a new approach to total flavonoid quantification, Talanta, 2013, 105, 192-203, DOI: 10.1016/ j.talanta.2012.11.072.

16 R. A. S. Alavarce, L. L. Saldanha, N. L. M. Almeida, V. C. Porto, A. L. Dokkedal and V. S. Lara, The beneficial effect of Equisetum giganteum L. against Candida biofilm formation: new approaches to denture stomatitis, J. Evidence-Based Complementary Altern. Med., 2015, 939625, DOI: $10.1155 / 2015 / 939625$.

17 M. Farinon, P. S. Lora, L. N. Francescato, et al., Effect of aqueous extract of giant horsetail (Equisetum giganteum L.) in antigen-induced arthritis, Open Rheumatol. J., 2013, 7, 129-133, DOI: 10.2174/1874312901307010129.

18 L. N. Francescato, D. A. Quinteros, S. Bordignon, V. L. Bassani and A. T. Henriques, Physicochemical characterization for quality control of Equisetum giganteum L., Lat. Am. J. Pharm., 2011, 30(6), 2383.

19 Y. Yayalaci, I. Celik and B. Bati, Hepatoprotective and antioxidant activity of linden (Tilia platyphyllos L.) infusion against ethanol-induced oxidative stress in rats, J. Membr. Biol., 2014, 247(2), 181-188, DOI: 10.1007/ s00232-013-9622-z.

20 P. Majer, S. Neugart, A. Krumbein, M. Schreiner and É. Hideg, Singlet oxygen scavenging by leaf flavonoids contributes to sunlight acclimation in Tilia platyphyllos, Environ. Exp. Bot., 2014, 100, 1-9, DOI: 10.1016/ j.envexpbot.2013.12.001.

21 A. Karioti, L. Chiarabini, A. Alachkar, M. F. Chehna, F. F. Vincieri and A. R. Bilia, HPLC-DAD and HPLC-ESI-MS analyses of Tiliae flos and its preparations, J. Pharm. Biomed. Anal., 2014, 100, 205-214, DOI: 10.1016/ j.jpba.2014.08.010.

22 A. H. P. Souza, R. C. G. Corrêa, L. Barros, et al., Phytochemicals and bioactive properties of Ilex paraguariensis: An in vitro comparative study between the whole plant, leaves and stems, Food Res. Int., 2015, 78, 286294, DOI: 10.1016/j.foodres.2015.09.032.

23 N. Martins, L. Barros, M. Dueñas, C. Santos-Buelga and I. C. F. R. Ferreira, Characterization of phenolic compounds and antioxidant properties of Glycyrrhiza glabra L. rhizomes and roots, RSC Adv., 2015, 5(34), 26991-26997, DOI: 10.1039/C5RA03963K.

24 O. Taofiq, R. C. Calhelha, S. Heleno, et al., The contribution of phenolic acids to the anti-inflammatory activity of mushrooms: Screening in phenolic extracts, individual parent molecules and synthesized glucuronated and methylated derivatives, Food Res. Int., 2015, 76, 821-827, DOI: 10.1016/j.foodres.2015.07.044. 
25 R. M. V. Abreu, I. C. F. R. Ferreira, R. C. Calhelha, et al., Anti-hepatocellular carcinoma activity using human HepG2 cells and hepatotoxicity of 6-substituted methyl 3- aminothieno [3,2-b] pyridine-2-carboxylate derivatives: In vitro evaluation, cell cycle analysis and QSAR studies, Eur. J. Med. Chem., 2011, 46, 5800-5806.

26 M. N. Clifford, K. L. Johnston, S. Knight and N. Kuhnert, Hierarchical scheme for LC-MSn identification of chlorogenic acids, J. Agric. Food Chem., 2003, 51(10), 2900-2911, DOI: $10.1021 /$ jf026187q.

27 G. Negri, D. Santi and R. Tabach, Flavonol glycosides found in hydroethanolic extracts from Tilia cordata, a species utilized as anxiolytics, Braz. J. Med. Plants, 2013, 15(2), 217-224.

28 G. Toker, M. Memişoğlu, E. Yeşilada and M. Aslan, Main flavonoids of Tilia argentea DESF. ex DC. Leaves, Turk. J. Chem., 2004, 28(6), 745-749.

29 E. Aguirre-Hernández, M. E. González-Trujano, A. L. Martínez, et al., HPLC/MS analysis and anxiolytic-like effect of quercetin and kaempferol flavonoids from Tilia americana var. mexicana, J. Ethnopharmacol., 2010, 127(1), 91-97, DOI: 10.1016/j.jep.2009.09.044.

30 F. Ieri, M. Innocenti, L. Possieri, S. Gallori and N. Mulinacci, Phenolic composition of "bud extracts" of Ribes nigrum L., Rosa canina L. and Tilia tomentosa M., J. Pharm. Biomed. Anal., 2015, 115, 1-9, DOI: 10.1016/ j.jpba.2015.06.004.

31 O. K. Kosakowska, K. Baczek, J. L. Przybył, et al., Intraspecific variability in the content of phenolic com- pounds, essential oil and mucilage of small-leaved lime (Tilia cordata Mill.) from Poland, Ind. Crops Prod., 2015, 78, 58-65, DOI: 10.1016/j.indcrop.2015.10.002.

32 C. Santos-Buelga, C. García-Viguera and F. A. TomásBarberán, On-line identification of flavonoids by HPLC coupled to diode array detection, in Methods in Polyphenol Analysis, Royal Societyof Chemistry, Cambridge, UK, 2003, pp. 92-127.

33 V. Exarchou, Y. C. Fiamegos, T. A. van Beek, C. Nanos and J. Vervoort, Hyphenated chromatographic techniques for the rapid screening and identification of antioxidants in methanolic extracts of pharmaceutically used plants, J. Chromatogr., A, 2006, 1112(1-2), 293-302, DOI: 10.1016/j. chroma.2005.11.077.

34 H. Correia, A. González-Paramás, M. T. Amaral, C. SantosBuelga and M. T. Batista, Characterization of polyphenols by HPLC-PAD-ESI/MS and antioxidant activity in Equisetum telmateia, Phytochem. Anal., 2005, 16(5), 380-387, DOI: 10.1002/pca.864.

35 L. Chen and Y.-H. Kang, Anti-inflammatory and antioxidant activities of red pepper (Capsicum annuum L.) stalk extracts: Comparison of pericarp and placenta extracts, J. Funct. Foods, 2013, 5(4), 1724-1731, DOI: 10.1016/j.jff.2013.07.018.

36 L. R. Silva, P. Valentão, J. Faria, et al., Phytochemical investigations and biological potential screening with cellular and non-cellular models of globe amaranth (Gomphrena globosa L.) inflorescences, Food Chem., 2012, 135(2), 756-763, DOI: 10.1016/j.foodchem.2012.05.015. 